\title{
Sternotomy for lung transplantation: a description of technique
}

\author{
Antonio Coppolino ${ }^{1 \#}$, Gita N. Mody ${ }^{2 \#}$, Daniel Rinewalt ${ }^{3}$, Emily Polhemus ${ }^{1}$, Steve K. Singh ${ }^{4}$, \\ Hari R. Mallidi ${ }^{1}$
}

\begin{abstract}
${ }^{1}$ Division of Thoracic Surgery, Department of Surgery, Brigham and Women's Hospital, Boston, MA, USA; ${ }^{2}$ Division of Cardiothoracic Surgery, Department of Surgery, University of North Carolina, Chapel Hill, NC, USA; ${ }^{3}$ Department of Cardiac Surgery, Brigham and Women's Hospital, Boston, MA, USA; ${ }^{4}$ Trillium Health Partners, University of Toronto, Toronto, Canada

"These authors contributed equally to this work.

Correspondence to: Antonio Coppolino, MD. Division of Thoracic Surgery, Department of Surgery, Brigham and Women's Hospital, Boston, MA, USA. Email: acoppolino@bwh.harvard.edu.
\end{abstract}

Submitted Jan 23, 2020. Accepted for publication Jan 24, 2020.

doi: $10.21037 /$ acs.2020.01.05

View this article at: http://dx.doi.org/10.21037/acs.2020.01.05

\section{Introduction}

Lung transplantation for end-stage lung disease (ESLD) has been described through posterolateral thoracotomy, anterior thoracotomy with and without transverse sternal division, and median sternotomy. Each incision provides distinct advantages but have key technical considerations with which lung transplant surgeons must be aware and facile. Utilization of a sternotomy approach may increase with time as the ESLD patient population ages, due to the need to perform cardiac valve replacement and/or coronary bypass concurrently in this group (1). Germaine technical considerations for lung transplantation via sternotomy are the routine use of cardiopulmonary bypass (CPB) with good drainage and venting both for capturing air and controlled reperfusion. We provide an illustrative case in order to describe our technique of sternotomy for lung transplantation.

\section{Clinical vignette}

A 69-year-old man with ESLD due to interstitial lung disease presented for bilateral lung transplantation. The patient had a history of GERD, diverticulosis and hyperlipidemia. Preoperative CT scan showed pulmonary fibrosis and a small chest cavity but no contraindications to proceeding with median sternotomy approach. Cardiac catheterization demonstrated angiographically normal coronary arteries and pulmonary hypertension with PA systolic pressures of $41 \mathrm{mmHg}$. The use of $\mathrm{CPB}$ was planned for intraoperative hemodynamic support. Bilateral orthotopic lung transplant (BOLT) proceeded through median sternotomy. The CPB run lasted 195 minutes. Warm ischemic time was 34 minutes for the right lung and 37 minutes for the left lung. The patient tolerated the procedure well.

\section{Surgical technique}

\section{Preparation}

The patient was intubated with a single lumen endotracheal tube. A radial arterial line was placed for hemodynamic monitoring. Swan Ganz catheter was deferred given lack of pulmonary hypertension in this case. The patient was positioned for median sternotomy. Preoperative antibiotics, steroids, and induction immunosuppressants are given according to institutional protocol.

\section{Exposition}

A median sternotomy was made, and a sternal retractor placed. After dissection of pleural adhesions, the bilateral hila were dissected, with careful retraction to avoid phrenic nerve injury. Full heparinization followed by bicaval and aortic cannulation for CPB was performed as hemodynamic support is required during retraction of the heart to allow adequate exposure. The bicaval strategy is important to ensure adequate drainage during the necessary retraction of the right hilum during explant and implant. Catheters were placed as vents in the aorta and main pulmonary artery. 


\section{Operation}

The right lung was explanted first. The superior pulmonary vein, inferior pulmonary vein, and pulmonary artery were separately encircled and divided with a vascular load stapler proximal to the lobar branches, but preserving adequate length for the anastomoses. The truncus arteriosus branch may be taken separately if the right pulmonary artery is enlarged. The bronchus was divided sharply and secured with stay sutures. Care was taken to ligate bronchial arteries and lymphatics. Left pneumonectomy then proceeded in a similar fashion. The hila were prepared by dissecting the vascular structures intrapericardially to provide additional length.

The donor lungs were then divided and prepared in routine fashion. The right lung was implanted first. The right mainstem anastomosis began at the junction of the cartilaginous and membranous airway, and the membranous posterior wall was completed first. The anastomosis was completed using the same stitch, bringing together the anterior cartilaginous portion. The right pulmonary veins were prepared by removing the staple line and dividing between the superior and inferior pulmonary vein, creating a single orifice. A clamp was not applied, as the aortic vent adequately deairs the left heart. Finally, the right pulmonary arterial anastomosis was completed. A vascular clamp may assist with preventing the right pulmonary artery from retracting behind the superior vena cava. We used double-armed Prolene sutures in a running fashion for all anastomoses. Left lung implantation proceeded in a similar fashion. The left pulmonary veins and often the left pulmonary artery can be anastomosed intrapericardially.

\section{Completion}

Vents were removed after deairing was completed. CPB was weaned, and the patient was decannulated. Bronchoscopy was done for toilet and to visually inspect the bronchial anastomoses. Chest drains were placed with attention to positioning the drains just over the diaphragm to prevent retained effusions. The sternotomy was closed in routine fashion.

\section{Comments}

\section{Clinical results}

The patient was extubated 7 hours following completion of the procedure. The postoperative course was uneventful. The patient was transferred from the intensive care unit on postoperative day 5 and to home on postoperative day 16. Routine bronchoscopy performed at 3 months demonstrated intact bronchial anastomoses bilaterally without evidence of stricture. Transbronchial lung biopsies demonstrated no evidence of acute cellular vascular or airway rejection.

\section{Advantages and caveats}

Lung transplantation via midline sternotomy can be done with excellent outcomes. Sternotomy can be used safely for most etiologies of ESLD. Review of our recent 18 month experience with 59 lung transplants via sternotomy showed a majority of cases were idiopathic pulmonary fibrosis (IPF) (37\%), followed by chronic obstructive pulmonary disease (10\%), bronchiectasis (10\%), cystic fibrosis (7\%), hypersensitivity pneumonitis (3\%), and bronchiolitis obliterans (2\%). Other etiologies of ESLD in patients undergoing sternotomy for lung transplantation at our institution included pulmonary fibrosis other than IPF, pulmonary arterial hypertension, and scleroderma (31\%). In all cases, the preoperative CT scan should be closely examined with attention to location of adhesions and enlarged calcified hilar lymph nodes, which may complicate the dissection. If increased exposure is required for these conditions, clamshell incision could be considered. In an analysis of our and others' surgical outcomes, case duration is routinely less with median sternotomy compared to bilateral thoracotomies (2). Sternotomy does require use of cardiopulmonary bypass to provide hemodynamic support, particularly for the left hilum exposure. While the routine use of cardiopulmonary bypass during lung transplantation is controversial, we believe the advantages provided by a less morbid incision outweigh the potential disadvantages.

\section{Acknowledgments}

Funding for this work was provided by the Brigham and Women's Hospital Department of Surgery.

\section{Footnote}

Conflicts of Interest: The authors have no conflicts of interest to declare.

\section{References}

1. Wigfield CH, Buie V, Onsager D. "Age" in lung 
transplantation: factors related to outcomes and other considerations. Curr Pulmonol Rep 2016;5:152-8.

2. Shudo Y, Rinewalt D, Lingala B, et al. Impact of Surgical

Cite this article as: Coppolino A, Mody GN, Rinewalt D, Polhemus E, Singh SK, Mallidi HR. Sternotomy for lung transplantation: a description of technique. Ann Cardiothorac Surg 2020;9(1):65-67. doi: 10.21037/acs.2020.01.05
Approach in Double Lung Transplantation: Median Sternotomy vs Clamshell Thoracotomy. Transplant Proc 2020. [Epub ahead of print]. 